\title{
Airway pressure release ventilation: a step forward?
}

\author{
Neil R. Maclntyre* ${ }^{*}$, Michael Gentile, John Davies, Stephen Bergin, Craig Rackley and Anne Mathews
}

(c) 2017 Springer-Verlag GmbH Germany, part of Springer Nature and ESICM

Dear Editor,

The trial by Zhou et al. [1] shows clinical benefit for airway pressure release ventilation (APRV) and is thus important. However, it comes from a single center and, because it was an unblinded study, the potential for bias in clinical decision-making during the trial is obvious. This does not mean bias was present, but these results must be replicated in multicenter studies before practice changes.

Control group management may have been suboptimal because of the use of the low $\mathrm{PEEP} / \mathrm{FiO}_{2}$ table from the original ARDS Network trial [2]. Studies subsequent to this trial (and a meta-analysis) suggest that tables using a higher PEEP structure in severe ARDS have outcome benefits over tables using lower PEEP [3].

The reported APRV plateau pressure (Pplat) is considerably lower than the Phigh. This makes little sense since there is no flow during much of Thigh, and thus Phigh by definition should equal Pplat. This discrepancy is likely explained by the reported APRV Pplat being calculated during a volume control breath with similar tidal volumes and set PEEP. This approach, however, ignores the inevitable auto-PEEP that would be present with APRV when using an early expiratory flow termination to set Tlow. This Pplat misrepresentation underscores how nuances of APRV may be underappreciated even by experienced users.
Before we invest resources to implement APRV on a large scale, assurances are needed to be sure this investment is justified. This study represents a significant step in this process but many more steps need to be taken.

\section{Compliance with ethical standards}

\section{Conflicts of interest}

On behalf of all authors, the corresponding author states that there is no conflict of interest.

Accepted: 28 November 2017

Published online: 11 December 2017

\section{References}

1. Zhou Y et al (2017) Early application of airway pressure release ventilation may reduce the duration of mechanical ventilation in acute respiratory distress syndrome. Intensive Care Med 43:1648-1659. https://doi. org/10.1007/s00134-017-4912-z

2. The ARDS Network (2000) Ventilation with lower tidal volumes as compared to traditional tidal volumes for acute lung injury and ARDS. N Engl Jed 342:1301-1308

3. Briel M et al (2010) Higher vs lower positive end-expiratory pressure in patients with acute lung injury and acute respiratory distress syndrome: systematic review and meta-analysis. JAMA 303:865-873 